\title{
Efficacy and safety of sorafenib in advanced renal cell carcinoma patients: Results from a long-term study
}

\author{
LIN YANG, LEI SHI, QIANG FU, HUIHUA XIONG, MENGXIAN ZHANG and SHIYING YU \\ Oncology Center, Tongji Hospital, Tongji Medical College, Huazhong Science and Technology University, \\ Wuhan 430030, P.R. China
}

Received November 16, 2011; Accepted January 19, 2012

DOI: $10.3892 / \mathrm{ol} .2012 .585$

\begin{abstract}
Sorafenib has been confirmed as an effective drug in advanced renal cell carcinoma (RCC). This study aimed to evaluate the long-term efficacy and safety of sorafenib in ethnic Chinese patients with advanced RCC, and to develop optimal treatment strategies for Asian patients. Between May 2006 and August 2011, 30 patients with advanced RCC were treated with sorafenib in the Oncology Center, Tongji Hospital. All 30 patients received continuous treatment with $400 \mathrm{mg}$ of sorafenib orally twice daily until disease progression or intolerable toxicities or mortality occurred. Dose reduction to $600 \mathrm{mg}$ daily or even less was required if toxicities of grade 3 or 4 occurred. Patients were assessed for tumor response, progression-free survival (PFS), overall survival (OS) and drug-related toxicities. The median follow-up time was 58 weeks (range, 12-270). Among the 30 patients, 1 patient had complete remission (CR 3.3\%), 4 patients had partial remission (PR 13.3\%), 19 patients had stable disease (SD 63.3\%) and 6 patients had disease progression (PD 20\%). The disease control rate (DCR, CR+PR+SD) was $80 \%$, the median PFS time was 14 months, and the median OS time was 16 months. Only 1 patient discon-
\end{abstract}

Correspondence to: Dr Shiying Yu, Oncology Center, Tongji Hospital, Tongji Medical College, Huazhong Science and Technology University, No. 1095 Jiefang Road, Wuhan 430030, P.R. China

E-mail:mdtjy@yahoo.cn; syyu@tjh.tjmu.edu.cn

Abbreviations: c-Kit, c-Kit protein; CR, complete remission; CTCAE, Common Terminology Criteria for Adverse events; DCR, disease control rate; ECOG, Eastern Cooperative Oncology Group; FDA, US Food and Drug Administration; Flt-3, FMS-like tyrosine kinase 3; HFSR, hand-foot skin reaction; MSKCC, Memorial Sloan-Kettering Cancer Center; MTD, maximum tolerated dose; ORR, objective response rate; OS, overall survival; PD, disease progression; PDGFR- $\beta$, platelet-derived growth factor receptor $\beta$; PFS, progression-free survival; PR, partial remission; RCC, renal cell carcinoma; RDI, relative dose intensity; RECIST, Response Evaluation Criteria in Solid Tumors; SD, stable disease; TARGET, Treatment Approaches in Renal Cancer Global Evaluation Trial; VEGFR, vascular endothelial growth factor receptor

Key words: sorafenib, renal cell carcinoma, targeted therapy, overall survival, toxicity, dosing tinued sorafenib treatment permanently due to severe toxicities. Dose reduction or interruption was required in 12 patients (40\%) who developed adverse events of grade 3 or 4 . Seven of these patients tolerated the dose of $600 \mathrm{mg}$ per day well, and experienced clinical benefit. The Kaplan-Meier method and log-rank test revealed that the Memorial Sloan-Kettering Cancer Center (MSKCC) status was a prognostic factor for PFS and OS in advanced RCC. The long-term efficacy and safety of sorafenib were confirmed in Chinese advanced RCC patients who showed an even greater benefit in PFS. The findings of this study indicate that a dose of $600 \mathrm{mg}$ instead of $400 \mathrm{mg}$ per day may be an optimal choice for Asian patients when a reduction of the initial dose is required.

\section{Introduction}

Renal cell carcinoma (RCC) is among the 10 most common types of malignant tumors, with an incidence rate that has increased by $2 \%$ per year for the past 65 years (1). Approximately 20 to $30 \%$ of patients with RCC have metastases at the time of diagnosis, and 20 to $40 \%$ of patients who undergo curative nephrectomy subsequently develop metastases (2). Advanced RCC is generally resistant to conventional chemotherapy and radiotherapy, and its response to cytokine treatment is less than $20 \%(3,4)$. In the past 10 years, targeted therapy utilizing tyrosine kinase inhibitors has proven efficacious and is widely used in advanced RCC.

Sorafenib tosylate (Nexavar, BAY 43-9006), an orally active multikinase inhibitor, is the first targeted drug approved for the treatment of advanced RCC by the US Food and Drug Administration (FDA) and the majority of Asian countries/ regions, including China. Sorafenib functions by blocking vascular endothelial growth factor receptors (VEGFR) 3 and 2, and platelet-derived growth factor receptor $\beta$ (PDGFR- $\beta$ ), as well as RAF-1, FMS-like tyrosine kinase 3 (Flt-3) and c-Kit protein (c-Kit) (5). The pivotal phase III multicenter Treatment Approaches in Renal Cancer Global Evaluation Trial (TARGET), a randomized double-blind placebo-controlled study, revealed the benefit of progression-free survival (PFS) (5.5 vs. 2.8 months) and overall survival (OS) (17.8 vs. 14.3 months) in sorafenib-treated patients, and established the efficacy and safety of sorafenib in advanced RCC $(4,6)$.

Certain molecular-targeted drugs, such as erlotinib and gefitinib, have been demonstrated to be significantly superior 
in Asian patients with non-small cell lung cancer compared with Caucasian patients, due to different tumor genetic characteristics (7), such as endothelial growth factor receptor (EGFR) exon 19 deletion and exon 21 L858R mutations (8-10). It is therefore reasonable to hypothesize that the efficacy and safety of sorafenib on advanced RCC may vary in different ethnic groups. As the TARGET study was mainly conducted in Caucasian patients, data on the administration of sorafenib in Asian patients, particularly Chinese patients, with advanced RCC is limited, especially for long-term evaluation of OS and the toxicities. The experience of using sorafenib clinically in Asian patients is also insufficient. This study aimed to document the experience of sorafenib treatment in Chinese patients with advanced RCC. Particular emphasis was placed on the OS of patients, long-term toxicities and the development of optimal treatment strategies for Asian patients.

\section{Materials and methods}

Patients and evaluation. From May 2006 to August 2011, 30 Chinese patients with advanced RCC were enrolled in this study. Inclusion criteria included: i) Age between 18 and 80 years; ii) histologically confirmed advanced RCC; iii) at least one measurable tumor lesion which was unresectable; iv) a life expectancy of at least 12 weeks; v) adequate liver, pancreatic and renal function, and a prothrombin time or partial-thromboplastin time of $<1.5$ times the upper limit of the normal range; vi) performance status of 0 to 2 on the basis of Eastern Cooperative Oncology Group (ECOG) criteria. All patients provided written informed consent prior to pretreatment evaluation.

Pretreatment evaluation included a complete history and physical examination, complete blood count, liver, pancreatic, and renal function tests, prothrombin time and partial-thromboplastin time tests, computed tomography (CT) scan of the chest, CT scan or magnetic resonance imaging (MRI) of the abdomen and pelvis and MRI of the brain. Emission CT for bone and PET/CT scan were optional. Patient characteristics are shown in Table I.

Treatment. The treatment protocol, based on the treatment recommendation approved by the US FDA for metastatic RCC, was approved by the Ethics Committee of our university. Patients received treatment with $400 \mathrm{mg}$ of sorafenib orally twice daily on a continuous dosing schedule, until disease progression or unacceptable toxicities or mortality occurred. Dose modification was permitted if grade of 3 or 4 toxicities defined by the Common Terminology Criteria for Adverse events (CTCAE) v3.0 occurred. Five levels were used in dose modification according to patient status: i) Level 1 (initial dose): $800 \mathrm{mg}$ per day, in doses of $400 \mathrm{mg}$ twice daily; ii) level 2: $600 \mathrm{mg}$ per day, $200 \mathrm{mg}$ in the morning and $400 \mathrm{mg}$ in the evening; iii) level 3: $400 \mathrm{mg}$ once per day; iv) level 4: $400 \mathrm{mg}$ once every 2 days; v) level 5: treatment interruptions. If adverse events resolved to a grade of 2 or less, the dose could be escalated to the previous level according to the discretion of the doctor.

Follow up. Radiological evaluation of the measurable tumor lesions was required every 8 weeks for the first year, and then
Table I. Baseline patient characteristics.

\begin{tabular}{|c|c|}
\hline Baseline clinical characteristics & No. of patients $(\%)$ \\
\hline \multicolumn{2}{|l|}{ Gender } \\
\hline Male & $21(70)$ \\
\hline Female & $9(30)$ \\
\hline \multicolumn{2}{|l|}{ Age, years } \\
\hline$<70$ & $26(86.7)$ \\
\hline$\geq 70$ & $4(13.3)$ \\
\hline \multicolumn{2}{|l|}{ ECOG performance status } \\
\hline $0-1$ & $21(70)$ \\
\hline$\geq 2$ & $9(30)$ \\
\hline \multicolumn{2}{|l|}{ Histology } \\
\hline Clear cell & $23(76.7)$ \\
\hline Non-clear cell & $4(13.3)$ \\
\hline Mixed & $3(10)$ \\
\hline \multicolumn{2}{|l|}{ Number of metastatic foci } \\
\hline 1 & $5(16.7)$ \\
\hline 2 & $8(26.7)$ \\
\hline$>2$ & $17(56.7)$ \\
\hline \multicolumn{2}{|l|}{ Sites of metastatic disease } \\
\hline Lung & $19(63.3)$ \\
\hline Liver & $5(16.7)$ \\
\hline Soft tissue & $5(16.7)$ \\
\hline \multicolumn{2}{|l|}{ Previous treatment } \\
\hline Cytokine & $13(43.3)$ \\
\hline Interleukin-2 & $7(23.3)$ \\
\hline Interferon & $8(26.7)$ \\
\hline Nephrectomy & $27(90)$ \\
\hline Radiotherapy & $9(30)$ \\
\hline Chemotherapy & $5(16.7)$ \\
\hline \multicolumn{2}{|l|}{ Duration of disease } \\
\hline$<1.5$ years & $17(56.7)$ \\
\hline$\geq 1.5$ years & $13(43.3)$ \\
\hline \multicolumn{2}{|l|}{ MSKCC category } \\
\hline Low risk & $11(36.7)$ \\
\hline Intermediate risk & $8(26.7)$ \\
\hline High risk & $11(36.7)$ \\
\hline
\end{tabular}

ECOG, Eastern Cooperative Oncology Group; MSKCC, Memorial Sloan-Kettering Cancer Center.

every 12 weeks thereafter in all patients. Tumor response was defined as complete remission (CR), partial remission (PR), stable disease (SD) and disease progression (PD) according to the Response Evaluation Criteria in Solid Tumors (RECIST). If the tumor response was evaluated as $\mathrm{CR}$ or $\mathrm{PR}$, confirmation was required 4 weeks later. The adverse events were evaluated every 2 to 4 weeks, including history collection, physical examination, complete blood count, liver, pancreatic and renal function tests, prothrombin time and partial-thromboplastin time tests. The toxicities were divided into grades 4 or 5 according to the CTCAE V3.0. 
Table II. Best tumor response rates.

\begin{tabular}{lcc}
\hline Response & No. of patients & Percentage $(\%)$ \\
\hline CR & 1 & 3.3 \\
PR & 4 & 13.3 \\
SD & 19 & 63.3 \\
PD & 6 & 20.0 \\
DCR & 24 & 80.0 \\
ORR & 5 & 16.7
\end{tabular}

$\mathrm{CR}$, complete remission; $\mathrm{PR}$, partial remission; $\mathrm{SD}$, stable disease; $\mathrm{PD}$, disease progression; DCR, disease control rate; ORR, objective response rate.

Statistical analysis. The disease control rate (DCR) was defined as the proportion of patients who achieved CR, PR and $\mathrm{SD}$, and the objective response rate (ORR) was defined as the proportion of patients who achieved CR and PR. The median PFS time was measured from the date of the start of sorafenib until the date of progression, or the date of the last follow-up visit if the patients were still alive without progression, or mortality. The median OS time was measured from the beginning of sorafenib until mortality, or the date of the last follow-up visit if the patients were still alive. The PFS and OS duration were calculated by the Kaplan-Meier method. Survival analyses were stratified by ECOG and MSKCC using the log-rank test.

Univariate analysis was used to evaluate whether these factors were capable of predicting the tumor response to sorafenib, including patients' age, ECOG status prior to treatment, MSKCC score, previous use or no use of cytokine therapy, presence or absence of lung or liver metastases, the time since diagnosis ( $<1.5$ or $\geq 1.5$ years) and the grade of hand-foot skin reaction (HFSR).

\section{Results}

Overall response. All 30 patients completed their evaluation of tumor response (Table II). The median follow-up time was 58 weeks (range, 12-270). In 18 patients (60\%), the follow-up time was more than 1 year. A total of 17 patients $(56.7 \%)$ received sorafenib as their first-line therapy, and 13 patients (43.3\%) received sorafenib as their second- or third-line therapy. Among the evaluable 30 patients, 1 patient had CR (3.3\%), 4 patients had PR (13.3\%), 19 patients had SD $(63.3 \%)$ and 6 patients had PD (20\%). The disease DCR was $80 \%$, and the ORR was $16.7 \%$.

PFS and OS. At the cut-off time of August 2011, 16 patients $(53.3 \%)$ had succumbed to the disease. Using the Kaplan-Meier method, the median OS was 16 months (95\% CI 10.227-21.773) (Fig. 1), and the median PFS was 14 months (95\% CI 0-31.704) (Fig. 2). Using the log-rank test, it was found that patients with different MSKCC scores had a statistical difference in PFS $(p=0.002)$ and OS time $(p=0.001)$, while patients with different ECOG status had no statistical difference in PFS time $(p=0.086)$ or OS time $(p=0.058)$.

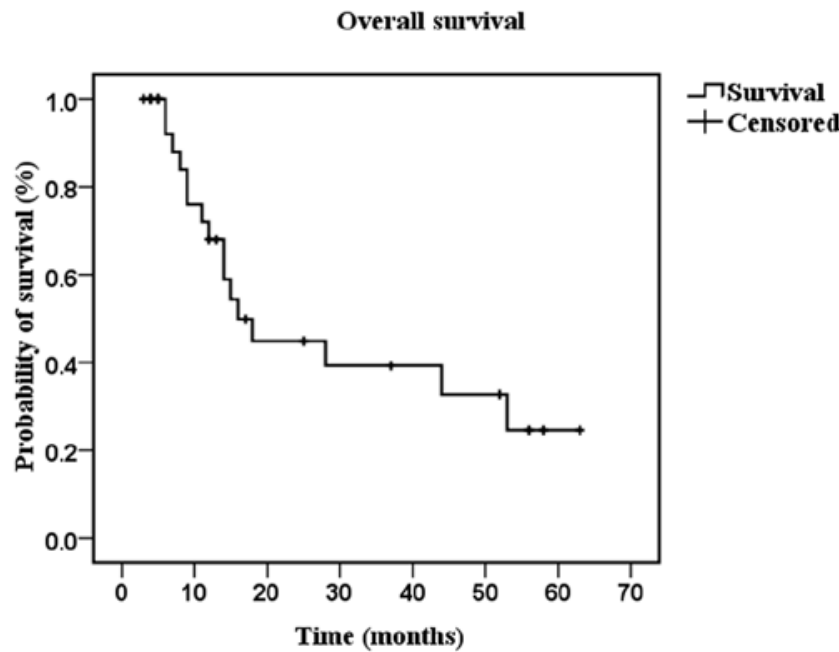

Figure 1. Overall survival is shown as calculated using the Kaplan-Meier method.

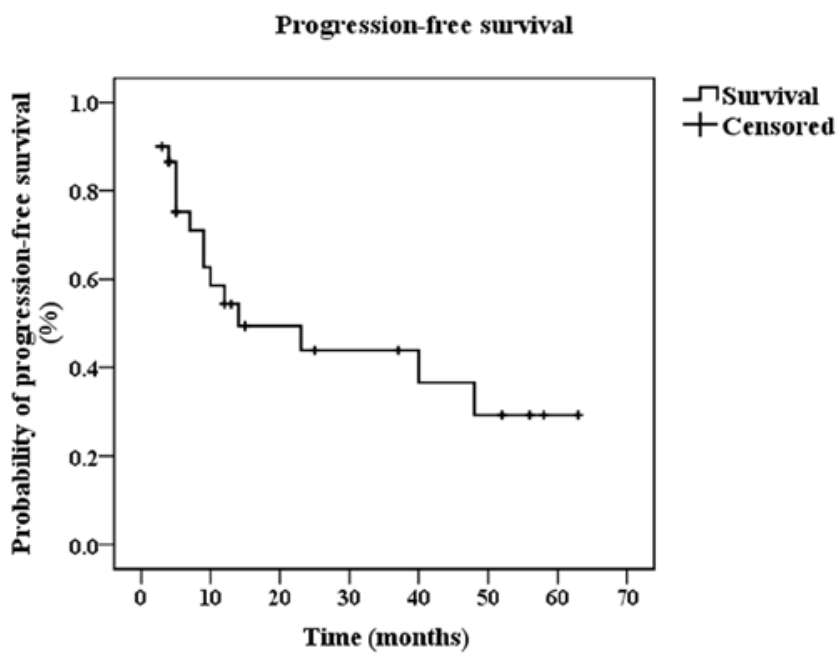

Figure 2. Progression-free survival is shown as calculated using the KaplanMeier method.

Adverse events. In the 30 patients, treatment with sorafenib was well tolerated. Only 1 patient discontinued sorafenib treatment due to intolerable adverse events, including rash of grade 3, oral cavity mucositis of grade 3 , diarrhea of grade 3 and gastrointestinal hemorrhage of grade 2. A total of 12 patients (40\%) reduced or interrupted their sorafenib treatment due to severe toxicities (predominantly HFSR of grade 3), among which 3 patients $(10 \%)$ recovered to the initial dose when the adverse events were alleviated. The common adverse events included HFSR in 18 patients $(60 \%)$, diarrhea in 10 patients $(33.3 \%)$, hypertension in 9 patients $(30 \%)$, rash in 9 patients $(30 \%)$, fatigue in 5 patients $(16.7 \%)$ and alopecia in 5 patients (16.7\%). The majority of adverse events were grade 1 or 2 , with the exception of HFSR (grade 3 in 8 patients, 26.7\%). These adverse events were reversible and clinically manageable. No cardiac ischemic/infarction adverse events were documented (Table III). In the 18 patients $(60 \%)$ who completed a follow-up time of more than 1 year, no additional or other severe adverse events were observed. 
Table III. Common adverse events.

\begin{tabular}{lccccc}
\hline Adverse events & Any grade $(\%)$ & Grade 1 $(\%)$ & Grade 2 $(\%)$ & Grade 3 $(\%)$ & Grade 4 (\%) \\
\hline HFSR & $18(60.0)$ & $3(10.0)$ & $7(23.3)$ & $8(26.7 \%)$ & $0(0)$ \\
Diarrhea & $10(33.3)$ & $3(10.0)$ & $6(20.0)$ & $1(3.3)$ & $0(0)$ \\
Fatigue & $5(16.7)$ & $4(13.3)$ & $1(3.3)$ & $0(0)$ & $0(0)$ \\
Rash & $9(30.0)$ & $3(10.0)$ & $5(16.7)$ & $1(3.3)$ & $0(0)$ \\
Hypertension & $9(30.0)$ & $4(13.3)$ & $4(13.3)$ & $1(3.3)$ & $0(0)$ \\
Alopecia & $5(16.7)$ & $3(10.0)$ & $2(6.6)$ & $0(0)$ & $0(0)$ \\
\hline
\end{tabular}

HFSR, hand-foot skin reaction.

Among the 12 patients who had reduced or interrupted their treatment of sorafenib, 7 patients had alleviation of the toxicity after their dose was modified to $600 \mathrm{mg}$ per day, a dose that was well tolerated by all 7 patients. Of these patients, 1 patient had PR, and the other 6 patients had SD. The best response lasted from 5 to 48 months (median time, 13 months).

Predictive factors of response to sorafenib. Results of the univariate analysis revealed that none of the factors was a significant predictive factor for tumor response to sorafenib, including patients' age, ECOG status prior to treatment, MSKCC score, previous use or no use of cytokine therapy, presence or absence of lung or liver metastases, the time since diagnosis and the grade of HFSR.

\section{Discussion}

At present, six targeted therapy agents have been approved by the US FDA for the treatment of advanced RCC, including sorafenib, sunitinib, pazopanib, temsirolimus, everolimus and bevacizumab in combination with interferon. However, only sorafenib and sunitinib were available in China at the end of 2009. The long-term efficacy and toxicity data from Asian patients are limited, which may be related to the low incidence of RCC in Asia (11). In the current study, we aimed to observe the long-term efficacy and safety of sorafenib in Chinese advanced RCC patients and to develop optimal treatment strategies for Asian patients.

The DCR obtained in our study was similar to that of previous studies $(4,12,13)$. Concerning the median PFS time, significant differences existed in various reports. In the TARGET study, the median PFS time was only 5.5 months, while our results showed the median PFS time to be 14 months. In another study on the treatment of sorafenib in 98 Chinese advanced RCC patients, the median PFS time was 60 weeks (13), which is very close to our data. In Japanese patients, PFS time was reported from 7.4 to 9 months $(12,14)$. We considered different ethnic background as an important factor leading to these differences. It appears that the Asian ethnic group, particularly Chinese patients, may experience more benefit of PFS from sorafenib treatment. Additional studies including a larger number of patients are necessary to verify these findings. Notably, the median OS time in this study was shorter than that in the TARGET study, despite the PFS benefit. The difference in patient characteristics was one reason for this observation. In the TARGET study, only patients with low or intermediate risk MSKCC score were enrolled, and the majority of them were evaluated with an ECOG status of 0 or 1 (1\% of patients with an ECOG status of 2) (4). However, in the current study, 11 patients (36.7\%) were evaluated with a high-risk MSKCC score, and 9 patients (30\%) with ECOG of 2. Furthermore, the follow-up therapy after failure of sorafenib involved best supportive treatment in this study, instead of dose escalation of sorafenib or other molecular-targeted therapy as in the case of Western countries, due to the limited available drugs and financial pressure in China.

The dose of sorafenib is considered to be positively correlated with its efficacy. In a series of phase I studies conducted in Western patients with solid tumors (15-18), $400 \mathrm{mg}$ twice daily was considered the maximum tolerated dose (MTD), and was recommended as the initial dose of sorafenib in follow-up studies. Later, it was found that following tumor progression at the recommended dose and schedule, tumor shrinkage was observed in $41.9 \%$ of 43 patients whose dose was increased to $600 \mathrm{mg}$ twice daily (19). A higher dose may be more effective as elevated concentrations result in greater inhibition of alternative target pathways as observed in RCC xenografts (20). It was also reported that a first one-month relative dose intensity (RDI) of not less than $50 \%$ predicted favorable PFS in sorafenib therapy for advanced RCC in Japanese patients (21). Considering these factors, when the initial dose was required to be reduced due to severe toxicities, we modified the daily dose from $800 \mathrm{mg}$ to $600 \mathrm{mg}$ instead of $400 \mathrm{mg}$, which had been widely recommended in the TARGET study and other follow-up studies. We found that the majority of the adverse events could be alleviated with a dose of $600 \mathrm{mg}$ daily, and the patients were able to receive long-term benefit from such treatment. The dose of $600 \mathrm{mg}$ per day may thus be a better choice than $400 \mathrm{mg}$ per day to balance the safety against the efficacy of sorafenib in Asian patients with advanced RCC. Since there is a high incidence of dose reduction in Asian patients due to sorafenib-related adverse events (11-13,22), further studies are required to investigate the feasibility of such therapy regimens which show preference for a daily dose of $600 \mathrm{mg}$ rather than $400 \mathrm{mg}$ when the reduction of the initial dose $(800 \mathrm{mg}$ per day) is required.

The Kaplan-Meier method and log-rank test revealed that the MSKCC score was prognostic for PFS and OS in advanced RCC, while ECOG status was not, which was similar to the results reported in the TARGET study (6). The MSKCC score 
was found to be relevant to the baseline VEGF levels, which were also prognostic factors for PFS and OS in advanced $\mathrm{RCC}$, but were not predictive factors of response to sorafenib. It was reported that patients receiving sorafenib doses at or close to the recommended $400 \mathrm{mg}$ bid dose, who experienced skin toxicity/diarrhea, had a significantly increased time to progression compared with patients without such toxicity $(\mathrm{P}<0.05)$ (23). However, in the current study, the results of the univariate analysis revealed that the grade of HFSR was not a predictive factor for tumor response to sorafenib. Compared with the TARGET study (4), our data indicated that the skin toxicity including HFSR in Chinese patients was much more frequent (60 vs. 33\%) and more severe (26.7 vs. $6 \%$ with grade $\geq 3$ ), as in the case of previous data obtained from Asia (11-13,22), and the resultant dose reduction may conceal the relevance between the grade of HFSR and the tumor response to sorafenib. A possible reason for the significant differences in the incidence of HFSR may include the difference in body surface area. In Asians, the median body surface area is much lower than that of Caucasians. Therefore, the optimal dose of $400 \mathrm{mg}$ bid for Caucasians may be extremely high for Asian patients. In our study, most patients with grade 3 of HFSR had good tolerance if the dose was reduced to $600 \mathrm{mg}$ per day. Another reason for the difference in incidence of HFSR may be the difference in genes or pharmacology. However, the related molecular mechanism remains to be elucidated.

The time of treatment with sorafenib ranged from 2 to 63 months, and 13 patients $(43.3 \%)$ received sorafenib for more than 1 year. In these patients, the incidence and severity of adverse events were similar to the other patients. The majority of adverse events occurred during the initial period of treatment with sorafenib. Neither unknown adverse events nor cumulative toxicity was observed during the long-term use of sorafenib, which was also confirmed by another report (24).

In conclusion, it was demonstrated that sorafenib was also safe and even more efficacious in Chinese advanced RCC patients with a median PFS time of 14 months. The dose of $600 \mathrm{mg}$ instead of $400 \mathrm{mg}$ per day may be an optimal treatment strategy for Asian patients when reduction of the initial dose was required. The MSKCC status prior to treatment with sorafenib was a prognostic factor for PFS and OS in advanced RCC.

\section{Acknowledgements}

This study was supported by China Charity Federation.

\section{References}

1. Pantuck AJ,Zisman A and Belldegrum AS: The changing natural history of renal cell carcinoma. J Urol 166: 1611-1623, 2001.

2. Gitlitz BJ and Figlin RA: Cytokine-based cherapy for metastatic renal cell cancer. Urol Clin North Am 30: 589-600, 2003

3. Motzer RJ, Bander NH and Nanus DM: Renal-cell carcinoma. N Engl J Med 335: 865-875, 1996.

4. Escudier B, Eisen T, Stadler WM, et al: TARGET Study Group. Sorafenib in advanced clear-cell renal-cell carcinoma. N Engl J Med 356: 125-134, 2007.

5. Wilhelm SM, Carter C, Tang L, et al: BAY43-9006 exhibits broad spectrum oral antitumor activity and targets the RAF/MEK/ ERK pathway and receptor tyrosine kinases involved in tumor progression and angiogenesis. Cancer Res 64: 7009-7109, 2004.
6. Escudier B, Eisen T, Stadier WM, et al: Sorafenib for treatment of renal cell carcinoma: Final efficacy and safety results of the phase III treatment approaches in renal cancer global evaluation trial. J Clin Oncol 27: 3312-3318, 2009.

7. Thatcher N, Chang A, Parikh P, et al: Gefitinib plus best supportive care in previously treated patients with refractory advanced non-small-cell lung cancer: results from a randomized, placebo-controlled, multicentre study (Iressa Survival Evaluation in Lung Cancer). Lancet 366: 1527-1537, 2005.

8. Tsao MS, Sakurada A, Cutz JC, et al: Erlotinib in lung cancer molecular and clinical predictors of outcome. N Engl J Med 353: 133-144, 2005.

9. Sequist LV, Marting RG, Spigel D, et al: First-line gefitinib in patients with advanced non-small-cell lung cancer harboring somatic EGFR mutations. J Clin Oncol 26: 2442-2449, 2008.

10. Hirsch FR and Bunn PA Jr: EGFR testing in lung cancer is ready for prime time. Lancet Oncol 10: 432-433, 2009.

11. Stafford HS, Saltzstein SL, Shimasaki S, Sanders C, Downs TM and Robins Sadler G. Racial/ethnic and gender disparities in renal cell carcinoma incidence and survival. J Urol 179: 1704-1708, 2008.

12. Tanigawa G, Kawashima A, Yamaguchi S, et al: Clinical outcome and prognostic factors of sorafenib in japanese patients with advanced renal cell carcinoma in general clinical practice. Jpn J Clin Oncol 41: 1265-1270, 2011.

13. Zhang H, Dong B, Lu JJ, et al: Efficacy of sorafenib on metastatic renal cell carcinoma in Asian patients: Results from a multicenter study. BMC Cancer 9: 249, 2009.

14. Akaza H, Tsukamoto T, Murai M, Nakajima $\mathrm{K}$ and Naito S: Phase II study to investigate the efficacy, safety, and pharmacokinetics of sorafenib in Japanese patients with advanced renal cell carcinoma. Jpn J Clin Oncol 37: 755-762, 2007.

15. Awada A, Hendlisz A, Gil T, et al: Phase I safety and pharmacokinetics of BAY 43-9006 administered for 21 days on/7 days off in patients with advanced, refractory solid tumours. Br J Cancer 92: 1855-1861, 2005.

16. Clark JW, Eder JP, Ryan D, Lathia C and Lenz HJ: Safety and pharmacokinetics of the dual action Raf kinase and vascular endothelial growth factor receptor inhibitor, BAY 43-9006, in patients with advanced, refractory solid tumors. Clin Cancer Res 11: 5472-5480, 2005.

17. Moore M, Hirte HW, Siu L, et al: Phase I study to determine the safety and pharmacokinetics of the novel Raf kinase and VEGFR inhibitor BAY 43-9006, administered for 28 days on/7 days off in patients with advanced, refractory solid tumors. Ann Oncol 16: 1688-1694, 2005.

18. Strumberg D, Richly H, Hilger RA, et al: Phase I clinical and pharmacokinetic study of the Novel Raf kinase and vascular endothelial growth factor receptor inhibitor BAY 43-9006 in patients with advanced refractory solid tumors. J Clin Oncol 23: 965-972, 2005.

19. Escudier B, Szczylik C, Hutson TE, et al: Randomized phase II trial of first-line treatment with sorafenib versus interferon Alfa-2a in patients with metastatic renal cell carcinoma. J Clin Oncol 27: 1280-1289, 2009.

20. Chang YS, Adnane J, Trail PA, et al: Sorafenib (BAY43-9006) inhibits tumor growth and vascularization and induces tumor apoptosis and hypoxia in RCC xenograft models. Cancer Chemother Pharmacol 59: 561-574, 2007.

21. Kawashima A, Takayama H, Arai Y, et al: One-month relative dose intensity (RDI) of not less than $50 \%$ predicts favourable progression-free survival in sorafenib therapy for advanced renal cell carcinoma in Japanese patients. Eur J Cancer 47: 1521-1526, 2011.

22. Iijima M, Fukino K, Adachi M, et al: Sorafenib-associated hand-foot syndrome in Japanese patients. J Dermatol 38: 261-266, 2011.

23. Strumberg D, Awada A, Hirte H, et al: Pooled safety analysis of BAY 43-9006 (sorafenib) monotherapy in patients with advanced solid tumours: Is rash associated with treatment outcome? Eur J Cancer 42: 548-556, 2006.

24. Hutson TE, Bellmunt J, Porta C, et al: Long-term safety of sorafenib in advanced renal cell carcinoma: follow-up of patients from phase III TARGET. Eur J Cancer 46: 2432-2440, 2010. 